Revista de Matemática: Teoría y Aplicaciones 2006 13(1) : 25-34

CIMPA - UCR - CCSS ISSN: 1409-2433

\title{
DEFORMACIONES ISOMÉTRICAS INFINITESIMALES DE UNA CLASE DE SUPERFICIES PEGADAS
}

\author{
Olman Trejos *
}

Recibido/Received: 30 Nov 2006 - Aceptado/Accepted: 30 Ago 2006

\begin{abstract}
Resumen
En este trabajo se demuestra la condición suficiente de rigidez de una superficie 4-dimensional pegada en el espacio 6-dimensional euclídeo. Esta superficie representa en sí, el producto de Riemann de dos superficies, cada una de las cuales se encuentra en el espacio 3-dimensional euclídeo y una de ellas es una superficie pegada.
\end{abstract}

Palabras clave: Producto de Riemann, superficie pegada, condición de yuxtaposición, reper, coreper, variedad, subvariedad, difeomorfismo, Deformaciones infinitesimales, deformación isométrica, deformación isométrica infinitesimal, campo de deformaciones infinitesimales.

\begin{abstract}
In this work the sufficient condition of rigidity of a stuck 4-dimensional surface in the Euclidean 6-dimensional space is demonstrated. This surface represents in himself, the product of Riemann of two surfaces, each one of which it is in the Euclidean 3dimensional space and one of them is a stuck surface
\end{abstract}

Keywords: Riemann product, stuck surface, infinitesimal condition of juxtaposition, to reper, to coreper, variety, subvariety, difeomorfism, deformations, isometric deformation, infinitesimal isometric deformation, field of infinitesimal deformations.

Mathematics Subject Classification: 32S25, 14H55.

\footnotetext{
*Sede Regional del Pacífico, Universidad de Costa Rica, Puntarenas, Costa Rica. E-Mail: otrejos@excite.com, otrejos@mail.ru
} 


\section{Introducción}

En el trabajo [1] se demostró que si las superficies $S_{1}$ y $S_{2}$ pertenecen a la clase $C^{3}$ y son regulares, entonces de su rigidez en el espacio $E^{m_{1}}$ y $E^{m_{2}}$ respectivamente surge la rigidez de la superficie $S$ en el espacio $E^{m_{1}+m_{2}}$. En el trabajo [4] la suavidad se redujo hasta la clase $C^{2}$. Desde luego surge la pregunta sobre la validez de este resultado en el caso cuando las superficies $S_{1}$ y $S_{2}$ no son regulares. En este trabajo demostraremos el teorema sobre la rigidez de la superficie $S$ en el caso cuando una de las superficies $S_{1}, S_{2}$ es pegada a trozos regulares.

El artículo está organizado de la siguiente manera; en la siguiente seccíon se describe como construir una superficie pegada, en la seccíon 1.2 se dan las fórmulas de Gauss y Veyngarten para superficies regulares, las condiciones de yuxtaposicion para superficies pegadas multidimensionales se presentan en la seccíon 1.3, en la 1.4 se construye un ejemplo de una superficie pegada tetradimensional en $E^{6}$ y se formula el teorema y finalmente en la seccíon 1.5 se detalla la demostración de dicho teorema.

\section{Deformaciones isométricas infinitesimales de una clase de superficies pegadas.}

\subsection{Construcción de una superficie pegada.}

Consideremos las variedades suaves, $X^{+}, X^{-}$, orientables, de Hausdorf clase $C^{\infty}$, que satisfacen el segundo axioma de numerabilidad de dimensiones $n^{+}$y $n^{-}$, con fronteras $\partial X^{+}$y $\partial X^{-}$(las cuales pueden ser vacías) y las subvariedades $\Gamma^{+}, \Gamma^{-} k$-dimensionales, con $1 \leq k<n^{ \pm}$, clase $C^{\infty}$ de las variedades $X^{+}$y $X^{-}$respectivamente, tales que existe $C^{\infty}$-difeomorfismo $\varphi: \Gamma^{+} \rightarrow \Gamma^{-}$(no se excluye el caso cuando $\Gamma^{ \pm} \cap \partial X^{ \pm} \neq \varnothing$ ). El difeomorfismo $\varphi$ lo llamaremos difeomorfismo de pegado. Sean $S^{+}, S^{-}$superficies en el espacio euclídeo $m$-dimensional $E^{m}$, definidas por las $C^{2}$-aplicaciones $\mathbf{r}^{+}: X^{+} \rightarrow E^{m}$, $\mathbf{r}^{-}: X^{-} \rightarrow E^{m}$, que satisfacen la condición

$$
\mathbf{r}^{+}(x)=\mathbf{r}^{-}(\varphi(x))
$$

para todo punto $x \in \Gamma^{+}$. El conjunto $S=S^{+} \cup S^{-}$lo llamaremos superficie, pegada por las superficies $F^{+}$y $F^{-}$a lo largo de la superficie $\gamma=\mathbf{r}^{+}\left(\Gamma^{+}\right)$. La superficie $\gamma$ la llamaremos superficie de pegado.

\subsection{Fórmulas de Gauss y Veyngarten para superficies regulares}

Consideremos la variedad $\Gamma$, suave $k$-dimensional, $k \geq 1$, de Hausdorf, orientable, que satisface el segundo axioma de numerabilidad y el espacio euclídeo $E^{m}, m$-dimensional. Sea $\mathbf{r}: \Gamma \rightarrow E^{m}$, una $C^{2}$-inmersión, $I(\mathbf{r})$ la métrica en $\Gamma$ inducida por esta inmersión. En el entorno de cada punto en $\Gamma$, está definido un $C^{1}$-correper local $\tau=\left(\tau^{i}\right)_{i=1}^{k}$, en el cual, la métrica $I(\mathbf{r})$ tiene forma normal: $I(\mathbf{r})=\sum_{i=1}^{k} \tau^{i} \otimes \tau^{i}$. Con $\phi_{j}^{i}$ vamos a representar las formas de relación de correper $\tau$, unívocamente definidas por las condiciones $d \tau^{i}=\sum_{j=1}^{k} \tau^{j} \wedge \phi_{j}^{i}, \quad \phi_{j}^{i}=-\phi_{i}^{j}$, donde $i, j=1, \ldots, k, d$ es el signo del diferencial externo. 
Representando con $\xi=\left(\xi^{i}\right)_{i=1}^{k}$ al reper local, que es dual al correper $\tau$ y suponiendo que $\mathbf{e}_{i}=\xi_{i}(\mathbf{r})$ para el diferencial de la inmersión $\mathbf{r}$, podemos escribir:

$$
d \mathbf{r}=\sum_{i=1}^{k} \tau^{i} \mathbf{e}_{i}, \quad i=1, \ldots, k .
$$

El campo $\mathbf{e}_{i}$ forma un $C^{1}$-reper local de la fibración tangencial de la superficie $\mathbf{r}(\Gamma)$ en el espacio $E^{m}$.

Sea $\nu=\left(\nu_{\sigma}\right)_{\sigma=1}^{p}, p=m-k$, un reper local ortonormal de la fibración normal de la superficie $\mathbf{r}(\Gamma)$. En cada punto $x$ del dominio del correper $\tau$, el conjunto de vectores $\mathbf{e}_{1}(x), \ldots, \mathbf{e}_{k}(x), \nu_{1}(x), \ldots, \nu_{p}(x)$ forman una base ortonormal del espacio $E^{m}$. El desarrollo de los diferenciales $d \mathbf{e}_{i}, d \nu_{\sigma}$ en esta base, nos lleva hacia las fórmulas de Gauss y de Veyngarten:

$$
\begin{aligned}
& d \mathbf{e}_{i}=\phi_{i}^{j} \mathbf{e}_{j}+\omega_{i}^{\sigma} \nu_{\sigma}, \\
& d \nu_{\sigma}=-\omega_{i}^{\sigma} \mathbf{e}_{i}+\varkappa_{\sigma}^{\rho} \nu_{\rho} .
\end{aligned}
$$

Aquí $\omega_{i}^{\sigma}$ son 1-formas, continuas, llamadas formas de inmersión, $\varkappa_{\sigma}^{\rho}$ son 1-formas, continuas, llamadas formas de torsión de la superficie $\mathbf{r}(\Gamma), i=1, \ldots, k, \sigma, \rho=1, \ldots, p$. Para las formas de inmersión y torsión, son válidas las siguientes igualdades:

$$
\begin{aligned}
& \omega_{i}^{\sigma} \wedge \tau^{i}=0, \\
& \varkappa_{\sigma}^{\rho}=-\varkappa_{\rho}^{\sigma} .
\end{aligned}
$$

\subsection{Condición de yuxtaposición para superficies pegadas multidimen- sionales}

Consideremos las deformaciones infinitesimales isométricas, de las superficies $S^{+}$y $S^{-}$ del punto 2.1, con campos de deformaciones isométricas $\delta \mathbf{r}^{+}$y $\delta \mathbf{r}^{-}$respectivamente, que satisfacen la siguiente condición:

$$
\delta \mathbf{r}^{-}(\varphi(x))=\delta \mathbf{r}^{+}(x) .
$$

Para todo punto $x \in \Gamma^{+}$. Al par $\delta \mathbf{r}=\left(\delta \mathbf{r}^{+}, \delta \mathbf{r}^{-}\right)$lo vamos a llamar el campo de deformaciones isométricas de la superficie pegada $S$. El campo de deformaciones infinitesimales isométricas $\delta \mathbf{r}$, lo llamaremos trivial si para él, $\delta \mathbf{r}^{+}=\Omega \cdot \mathbf{r}^{+}+\omega, \delta \mathbf{r}^{-}=\Omega \cdot \mathbf{r}^{-}+\omega$, donde $\Omega$ es un bivector constante del cuadrado externo $\bigwedge^{2} E^{m}$ del espacio $E^{m}$, $\omega$ es un vector arbitrario del espacio $E^{m}$, el punto representa el producto interno de un bivector por un vector. La superficie pegada $S$ se llama rígida, si todo campo de deformaciones infinitesimales isométricas de $S$ es trivial.

Representemos con $\mathbf{V}^{+}$y $\mathbf{V}^{-}$a los campos de rotación de las superficies $S^{+}$y $S^{-}$ respectivamente. Ellos unívocamente definen las deformaciones infinitesimales isométricas de estas superficies con las siguientes igualdades:

$$
d \delta \mathbf{r}^{ \pm}=\mathbf{V}^{ \pm} \cdot d \mathbf{r}^{ \pm}
$$


De las condiciones de pegado (1) y (5), surge que en la variedad $\Gamma^{+}$, tienen lugar las igualdades $d \mathbf{r}^{+}(x)=d \mathbf{r}^{-}(\varphi(x))$ y $d \delta \mathbf{r}^{+}(x)=d \delta \mathbf{r}^{-}(\varphi(x))$. De aquí y de (6) tenemos:

$$
\left(\mathbf{V}^{+}(x)-\mathbf{V}^{-}(\varphi(x))\right) \cdot d \mathbf{r}(x)=0,
$$

donde $\mathbf{r}(x)=\mathbf{r}^{+}(x)$ para todo punto $x$ en $\Gamma^{+}$.

De las ecuaciones (2) y (7), llegamos a la igualdad:

$$
\left(\mathbf{V}^{+}(x)-\mathbf{V}^{-}(\varphi(x))\right) \cdot \mathbf{e}_{i}=0 .
$$

De tal forma, la diferencia $\mathbf{V}^{+}-\mathbf{V}^{-}=\mathbf{V}^{+}(x)-\mathbf{V}^{-}(\varphi(x))$ la podemos escribir:

$$
\mathbf{V}^{+}-\mathbf{V}^{-}=\frac{1}{2} A^{\tau \sigma}\left[\nu_{\tau}, \nu_{\sigma}\right]
$$

donde $\left[\nu_{\tau}, \nu_{\sigma}\right] \in \bigwedge^{2} E^{m}$ y las funciones $A^{\tau \sigma}$ satisfacen la condición $A^{\tau \sigma}=-A^{\sigma \tau}$. Derivando (8), en $\Gamma^{+}$obtenemos:

$$
d_{\Gamma_{\Gamma^{+}}} \mathbf{V}^{+}-d_{\Gamma_{\Gamma^{+}}} \mathbf{V}^{-}=\frac{1}{2} d A^{\tau \sigma}\left[\nu_{\tau}, \nu_{\sigma}\right]+A^{\sigma \tau}\left[d \nu_{\sigma}, \nu_{\tau}\right]
$$

Se sabe que el diferencial del campo de rotación se puede representar también de la forma:

$$
d_{\left.\right|_{\Gamma^{+}}} \mathbf{V}^{ \pm}=\delta \omega_{\left.\alpha\right|_{\Gamma^{+}} ^{ \pm}}^{ \pm}\left[\mathbf{e}_{\alpha}^{ \pm}, \nu_{\sigma}^{ \pm}\right]-\frac{1}{2} \delta \varkappa_{\left.\rho\right|_{\Gamma^{+}}}^{ \pm \tau}\left[\nu_{\tau}^{ \pm}, \nu_{\rho}^{ \pm}\right]
$$

donde $\left(\mathbf{e}_{\alpha}^{ \pm}\right)_{\alpha=1}^{n^{ \pm}}$y $\left(\nu_{\sigma}^{ \pm}\right)_{\sigma=1}^{m-n^{ \pm}}$son los reperes tangencial y normal de las superficies $F^{ \pm}$, $\left.\delta \omega^{ \pm \sigma}\right|_{\Gamma^{+}}$son las variaciones de las formas de inmersión de las superficies $F^{ \pm}$, restringidas en $\Gamma^{+}, \sigma=1, \ldots, m-n^{ \pm}, \quad \alpha=1, \ldots, n^{ \pm}$. Ver $[2,3]$.

Utilizando en (9) las fórmulas de Veyngarten y las expresiones para $d_{\left.\right|_{\Gamma^{+}}} \mathbf{V}^{ \pm}$, vamos a tener:

$$
\begin{aligned}
& \delta \omega^{+\sigma}{ }_{\alpha \Gamma_{\Gamma^{+}}}\left[\mathbf{e}_{\alpha}^{+}, \nu_{\sigma}^{+}\right]-\frac{1}{2} \delta \varkappa_{\left.\rho\right|_{\Gamma^{+}}}^{+\tau}\left[\nu_{\tau}^{+}, \nu_{\rho}^{+}\right]- \\
& -\delta \omega^{-\sigma}{ }_{\left.\alpha\right|_{\Gamma^{+}}}\left[\mathbf{e}_{\alpha}^{-}, \nu_{\sigma}^{-}\right]+\frac{1}{2} \delta \varkappa_{\left.\rho\right|_{\Gamma^{+}}}^{-\tau}\left[\nu_{\tau}^{-}, \nu_{\rho}^{-}\right]= \\
& =\frac{1}{2} d A^{\tau \sigma}\left[\nu_{\tau}, \nu_{\sigma}\right]-A^{\sigma \tau} \omega_{i}^{\sigma}\left[\mathbf{e}_{i}, \nu_{\tau}\right]+A^{\sigma \tau} \varkappa_{\sigma}^{\theta}\left[\nu_{\theta}, \nu_{\tau}\right] .
\end{aligned}
$$

Multipliquemos la última igualdad en forma escalar por el bivector $\left[\mathbf{e}_{\eta}, \nu_{\xi}\right]$. Sin pérdida de generalidad, podemos suponer que los primeros $k$ campos del reper ortonormal de la fibración tangencial de $S^{+}$y $S^{-}$, coinciden con los campos $\mathbf{e}_{1}, \ldots, \mathbf{e}_{k}$ del reper ortonormal de la fibración tangencial de la superficie $\gamma$. Tomando en cuenta que $\left[\nu_{\tau}^{+}, \nu_{\sigma}^{+}\right] \cdot\left[\mathbf{e}_{\eta}, \nu_{\xi}\right]=0$, $\left[\mathbf{e}_{i}, \nu_{\tau}\right] \cdot\left[\mathbf{e}_{\eta}, \nu_{\xi}\right]=\delta_{i \eta} \delta_{\tau \xi},\left[\mathbf{e}_{\beta}^{+}, \nu_{\sigma}^{+}\right] \cdot\left[\mathbf{e}_{\eta}, \nu_{\xi}\right]=\delta_{\beta \eta}\left(\nu_{\sigma}^{+}, \nu_{\xi}\right)$, donde $\delta_{i j}$ son los símbolos de Kronecker, $(*, *)$ es producto escalar en $E^{m}$, la igualdad (10) la podemos escribir de la siguiente forma:

$$
\begin{aligned}
& \delta \omega_{\left.\eta\right|_{\Gamma^{+}} ^{+}}^{+\lambda}\left(\nu_{\lambda}^{+}, \nu_{\xi}\right)-\delta \omega_{\left.\eta\right|_{\Gamma^{+}}}^{-\rho}\left(\nu_{\rho}^{-}, \nu_{\xi}\right)=-A^{\sigma \xi} \omega_{\eta}^{\sigma}, \\
& \eta=1, \ldots, k \xi=1 \ldots, m-k .
\end{aligned}
$$


Ahora multipliquemos a (10) de forma escalar por el bivector $\left[\nu_{\alpha}, \nu_{\rho}\right]$. Después de las transformaciones obtenemos la igualdad:

$$
\begin{aligned}
& \delta \omega_{\left.\beta\right|_{\Gamma^{+}}}^{\sigma}\left(\left(\mathbf{e}_{\beta}^{+}, \nu_{\alpha}\right)\left(\nu_{\sigma}^{+}, \nu_{\rho}\right)-\left(\mathbf{e}_{\beta}^{+}, \nu_{\rho}\right)\left(\nu_{\sigma}^{+}, \nu_{\alpha}\right)\right)- \\
& -\delta \omega_{\left.\beta\right|_{\Gamma^{+}}}^{-\sigma}\left(\left(\mathbf{e}_{\beta}^{-}, \nu_{\alpha}\right)\left(\nu_{\sigma}^{-}, \nu_{\rho}\right)-\left(\mathbf{e}_{\beta}^{-}, \nu_{\rho}\right)\left(\nu_{\sigma}^{-}, \nu_{\alpha}\right)\right)- \\
& -\delta \varkappa_{\left.\sigma\right|_{\Gamma^{+}} ^{+}}^{+\tau}\left(\nu_{\tau}^{+}, \nu_{\alpha}\right)\left(\nu_{\sigma}^{+}, \nu_{\rho}\right)+\delta \varkappa_{\left.\sigma\right|_{\Gamma^{+}}}^{-\tau}\left(\nu_{\tau}^{-}, \nu_{\alpha}\right)\left(\nu_{\sigma}^{-}, \nu_{\rho}\right)= \\
& =d A^{\alpha \beta}+A^{\sigma \rho} \varkappa_{\sigma}^{\alpha}-A^{\sigma \alpha} \varkappa_{\sigma}^{\rho}, \quad \alpha, \rho=1, \ldots, m-k .
\end{aligned}
$$

Las ecuaciones (11) y (12) las llamaremos las condiciones de yuxtaposición en la superficie de pegado $\gamma$.

\subsection{Rigidez de una superficie pegada}

Construiremos un ejemplo de una superficie pegada tetradimensional en $E^{6}$. Sea $M^{+}$y $M^{-}$regiones en la variedad bidimensional $M$ tal que $M=M^{+} \cup M^{-}$y $\bar{M}^{+} \cap \bar{M}^{-}=L$, donde $L$ es una subvariedad suave de la variedad $M$. Representemos con $E_{1}^{3}$ al espacio euclídeo tridimensional y definimos a $C^{2}$-inmersiones $\mathbf{r}_{1}^{+}: M^{+} \rightarrow E_{1}^{3}, \mathbf{r}_{1}^{-}: M^{-} \rightarrow E_{1}^{3}$, que satisfacen la siguiente condición $\mathbf{r}_{1}^{+}(x)=\mathbf{r}_{1}^{-}(x)$ para todo punto $x \in L$. Las imágenes $F_{1}^{+}=\mathbf{r}_{1}^{+}(M)$ y $F_{1}^{-}=\mathbf{r}_{1}^{-}(M)$ se llaman superficies, localizadas en $E_{1}^{3}$ y su unión $F_{1}=$ $F_{1}^{+} \cup F_{1}^{-}$es la superficie, obtenida al pegar a $F_{1}^{+}$y $F_{1}^{-}$a lo largo de la curva $\gamma=\mathbf{r}_{1}^{+}(L)$. Sea $Y$ una variedad bidimensional y $\mathbf{r}_{2}: Y \rightarrow E_{2}^{3}$ su $C^{2}$-inmersión en el espacio tridimensional euclídeo $E_{2}^{3}$, que tiene como imagen a $F_{2}=\mathbf{r}_{2}(Y)$, la cual representa a una superficie regular en $E_{2}^{3}$.

Usando notaciones del punto 2.1, sean $\Gamma^{+}=\Gamma^{-}=\Gamma=L \times Y, X^{+}=M^{+} \times Y$, $X^{-}=M^{-} \times Y$. En calidad de difeomorfismo de pegado, tomemos la aplicación identidad $i d: \Gamma \rightarrow \Gamma$. Sean $\mathbf{r}^{+}: X^{+} \rightarrow E^{6}, \quad \mathbf{r}^{-}: X^{-} \rightarrow E^{6} C^{2}$-inmersiones. Estas inmersiones satisfacen la siguiente condición $\mathbf{r}^{+}(x)=\mathbf{r}^{-}(x)$ para todo punto $x \in \Gamma$. Las imágenes $F^{+}=\mathbf{r}^{+}\left(X^{+}\right)$y $F^{-}=\mathbf{r}^{-}\left(X^{-}\right)$se llaman superficies tetradimensionales, localizadas en $E^{6}$. La unión de éstas $F=F^{+} \cup F^{-}$es una superficie, pegada por $F^{+}$y $F^{-}$a lo largo de la superficie tridimensional $S=\mathbf{r}^{+}(\Gamma)$.

Para la superficie $F$, es válido el siguiente teorema

Teorema 2.1 Si las superficies $F_{1}$ y $F_{2}$ son rígidas en los espacios $E_{1}^{3}$ y $E_{2}^{3}$ respectivamente, entonces $F$ es rígida en el espacio $E^{6}$.

\subsection{Demostración.}

2.5.1. En cada punto de las superficies $F_{1}^{+}$y $F_{1}^{-}$en $E_{1}^{3}$, están definidos los reperes tangencial y normal. Representemos con $\mathbf{e}_{1}^{+}\left(u^{1}, u^{2}\right), \mathbf{e}_{2}^{+}\left(u^{1}, u^{2}\right)$ y $\mathbf{e}_{1}^{-}\left(u^{1}, u^{2}\right), \mathbf{e}_{2}^{-}\left(u^{1}, u^{2}\right)$ los reperes tangenciales de las superficies respectivamente y con $\nu_{1}^{+}\left(u^{1}, u^{2}\right)$ y $\nu_{1}^{-}\left(u^{1}, u^{2}\right)$ los reperes normales, donde $u^{1}, u^{2}$ son coordenadas locales en la variedad $M$. 
Con $\mathbf{e}_{1}\left(u^{1}\right), \nu_{1}\left(u^{1}\right), \nu_{2}\left(u^{1}\right)$ vamos a representar a los reperes tangencial y normal de la línea de pegado $\gamma$, con $\mathbf{e}_{3}\left(u^{3}, u^{4}\right), \mathbf{e}_{4}\left(u^{3}, u^{4}\right), \quad \nu_{3}\left(u^{3}, u^{4}\right)$ a los reperes tangencial $\mathrm{y}$ normal de la superficie $F_{2}$ en $E_{2}^{3}$, donde $u^{3}, u^{4}$ son coordenadas locales en la variedad $Y$.

Los vectores $\mathbf{e}_{1}^{+}\left(u^{1}, u^{2}\right), \mathbf{e}_{2}^{+}\left(u^{1}, u^{2}\right), \quad \mathbf{e}_{3}\left(u^{3}, u^{4}\right), \mathbf{e}_{4}\left(u^{3}, u^{4}\right), \quad \nu_{1}^{+}\left(u^{1}, u^{2}\right), \quad \nu_{3}\left(u^{3}, u^{4}\right) \mathrm{y}$ $\mathbf{e}_{1}^{-}\left(u^{1}, u^{2}\right), \quad \mathbf{e}^{-}\left(u^{1}, u^{2}\right), \quad \mathbf{e}_{3}\left(u^{3}, u^{4}\right), \quad \mathbf{e}_{4}\left(u^{3}, u^{4}\right), \nu_{1}^{-}\left(u^{1}, u^{2}\right)$, forman los reperes tangenciales y normales de las superficies $F^{+}$y $F^{-}$en $E^{6}$ respectivamente, los vectores $\mathbf{e}_{1}\left(u^{1}\right)$, $\mathbf{e}_{3}\left(u^{3}, u^{4}\right), \quad \mathbf{e}_{4}\left(u^{3}, u^{4}\right), \quad \nu_{1}\left(u^{1}\right), \nu_{2}\left(u^{1}\right), \quad \nu_{3}\left(u^{3}, u^{4}\right)$ son los reperes tangencial y normal de la superficie de pegado $S \subset E^{6}$.

En adelante vamos suponer que en $\gamma, \mathbf{e}_{1}\left(u^{1}\right)=\mathbf{e}_{1}^{+}\left(u^{1}, u^{2}\right)=\mathbf{e}_{1}^{-}\left(u^{1}, u^{2}\right)$.

Consideremos las deformaciones infinitesimales isométricas de la superficie $F_{1}$ en $E_{1}^{3}$. Escribiremos las condiciones de yuxtaposición (11) y (12) en la línea de pegado $\gamma$ :

$$
\begin{aligned}
& \delta \omega^{+}{ }_{\left.1\right|_{L}}^{1}\left(\nu_{1}^{+}, \nu_{1}\right)-\delta \omega^{-1}{ }_{\left.1\right|_{L}}\left(\nu_{1}^{-}, \nu_{1}\right)=-\tilde{A}^{21} \omega_{1}^{2} \\
& \delta \omega^{+}{ }_{\left.1\right|_{L}}^{1}\left(\nu_{1}^{+}, \nu_{2}\right)-\delta \omega^{-1}{ }_{\left.1\right|_{L}}\left(\nu_{1}^{-}, \nu_{2}\right)=-\tilde{A}^{12} \omega_{1}^{1} \\
& \delta \omega^{+}{ }_{\left.2\right|_{L}}^{1}\left(\left(\mathbf{e}_{2}^{+}, \nu_{2}\right)\left(\nu_{1}^{+}, \nu_{2}\right)-\left(\mathbf{e}_{2}^{+}, \nu_{2}\right)\left(\nu_{1}^{+}, \nu_{1}\right)\right)- \\
& -\delta \omega^{-1}{ }_{\left.2\right|_{L}}\left(\left(\mathbf{e}_{2}^{-}, \nu_{2}\right)\left(\nu_{1}^{-}, \nu_{2}\right)-\left(\mathbf{e}_{2}^{-}, \nu_{2}\right)\left(\nu_{1}^{-}, \nu_{1}\right)\right)=d \tilde{A}^{12}
\end{aligned}
$$

De las condiciones de rigidez de la superficie $F_{1}$ en $E_{1}^{3}$ surge, que $\delta \omega^{ \pm}{ }_{\left.1\right|_{L}}^{1}=0, \quad \delta \omega^{+}{ }_{\left.2\right|_{L}}^{1}=0$, $\tilde{A}^{12}=0[2]$.

2.5.2 Consideremos las deformaciones infinitesimales isométricas de la superficie $F$ en $E^{6}$. Las condiciones de yuxtaposición en la superficie de pegado $S$ se verán de la siguiente forma:
1) $\delta \omega^{+}{ }_{\left.1\right|_{\Gamma}}^{1}\left(\nu_{1}^{+}, \nu_{1}\right)-\delta \omega^{-1}{ }_{\left.1\right|_{\Gamma}}\left(\nu_{1}^{-}, \nu_{1}\right)=-A^{21} \omega_{1}^{2}$
2) $\delta \omega^{+}{ }_{\left.1\right|_{\Gamma}}^{1}\left(\nu_{1}^{+}, \nu_{2}\right)-\left.\delta \omega^{-1}\right|_{\Gamma}\left(\nu_{1}^{-}, \nu_{2}\right)=-A^{12} \omega_{1}^{1}$
3) $\delta \omega^{+}{ }_{\left.1\right|_{\Gamma}}^{2}-\delta \omega^{-2}{ }_{\left.1\right|_{\Gamma}}=-A^{13} \omega_{1}^{1}-A^{23} \omega_{1}^{2}$
4) $\delta \omega^{+}{ }_{\left.2\right|_{\Gamma}}^{1}\left(\nu_{1}^{+}, \nu_{1}\right)-\delta \omega_{\left.2\right|_{\Gamma}}^{-1}\left(\nu_{1}^{-}, \nu_{1}\right)=-A^{31} \omega_{2}^{3}$
5) $\delta \omega^{+}{ }_{2 \mid \Gamma}^{1}\left(\nu_{1}^{+}, \nu_{2}\right)-\delta \omega^{-1}{ }_{\left.2\right|_{\Gamma}}\left(\nu_{1}^{-}, \nu_{2}\right)=-A^{32} \omega_{2}^{3}$
6) $\delta \omega^{+2}{ }_{\left.2\right|_{\Gamma}}^{2}-\delta \omega_{\left.2\right|_{\Gamma}}^{-2}=0$
7) $\delta \omega^{+}{ }_{\left.3\right|_{\Gamma}}^{1}\left(\nu_{1}^{+}, \nu_{1}\right)-\delta \omega^{-1}{ }_{\left.3\right|_{\Gamma}}\left(\nu_{1}^{-}, \nu_{1}\right)=-A^{31} \omega_{3}^{3}$ 


$$
\begin{aligned}
& \text { 8) } \delta \omega^{+}{ }_{\left.3\right|_{\Gamma}}^{1}\left(\nu_{1}^{+}, \nu_{2}\right)-\delta \omega^{-1}{ }_{\left.3\right|_{\Gamma}}^{-}\left(\nu_{1}^{-}, \nu_{2}\right)=-A^{32} \omega_{3}^{3} \\
& \text { 9) } \quad \delta \omega^{+2}{ }_{\left.3\right|_{\Gamma}}^{2}-\left.\delta \omega^{-2}\right|_{\Gamma}=0 \\
& \text { 10) } \delta \omega^{+}{ }_{2||_{\Gamma}}^{1}\left(\left(\mathbf{e}_{2}^{+}, \nu_{2}\right)\left(\nu_{1}^{+}, \nu_{2}\right)-\left(\mathbf{e}_{2}^{+}, \nu_{2}\right)\left(\nu_{1}^{+}, \nu_{1}\right)\right)- \\
& -\delta \omega_{\left.2\right|_{\Gamma}}^{-1}\left(\left(\mathbf{e}_{2}^{-}, \nu_{2}\right)\left(\nu_{1}^{-}, \nu_{2}\right)-\left(\mathbf{e}_{2}^{-}, \nu_{2}\right)\left(\nu_{1}^{-}, \nu_{1}\right)\right)=d A^{12} \\
& \text { 11) } \delta \omega^{+2}{ }_{\left.2\right|_{\Gamma}}^{2}\left(\mathbf{e}_{2}^{+}, \nu_{1}\right)-\delta \omega_{\left.2\right|_{\Gamma}}^{-2}\left(\mathbf{e}_{2}^{-}, \nu_{1}\right)- \\
& -\delta \varkappa_{\left.2\right|_{\Gamma} ^{+}}^{+1}\left(\nu_{1}^{+}, \nu_{1}\right)+\delta \varkappa_{\left.2\right|_{\Gamma}}^{-1}\left(\nu_{1}^{-}, \nu_{1}\right)=d A^{13} \\
& \text { 12) } \delta \omega^{+}{ }_{\left.2\right|_{\Gamma}}^{2}\left(\mathbf{e}_{2}^{+}, \nu_{2}\right)-\left.\delta \omega^{-2}\right|_{\Gamma}\left(\mathbf{e}_{2}^{-}, \nu_{2}\right)- \\
& -\delta \varkappa_{\left.2\right|_{\Gamma}}^{+1}\left(\nu_{1}^{+}, \nu_{2}\right)+\delta \varkappa_{\left.2\right|_{\Gamma}}^{-1}\left(\nu_{1}^{-}, \nu_{2}\right)=d A^{23} \text {. }
\end{aligned}
$$

Aquí las ecuaciones $1-9$ son obtenidas de la condición (11) y las ecuaciones $10-12$ de la (12).

Observemos que la primera, segunda y novena condiciones de yuxtaposición en $S \subset E^{6}$, coinciden con las condiciones de yuxtaposicón en $\gamma \subset E_{1}^{3}$. Por lo tanto, $\delta \omega^{ \pm}{ }_{\left.1\right|_{\Gamma}}^{1}=0$, $\delta \omega^{ \pm}{ }_{\left.2\right|_{\Gamma}}^{1}=0, A^{12}=0$. Aprovechando las ecuaciones cuarta y quinta y las condiciones de yuxtaposición en $\gamma$, vamos tener: $A^{31} \omega_{2}^{3}=0, A^{32} \omega_{2}^{3}=0$, de donde surge que $A^{13}=A^{23}=$ 0 . De aquí y de la ecuación (8) en $S$, obtenemos que $\mathbf{V}^{+}=\mathbf{V}^{-}$.

2.5.3. Todos los conceptos de este punto se darán para las superficies $F_{1}^{+} \subset E_{1}^{3}$ y $F^{+} \subset E^{6}$. Los resultados obtenidos para ellas serán válidos también para las superficies $F_{1}^{-} \subset E_{1}^{3} \quad F^{-} \subset E^{6}$.

Escribiremos el principal sistema de las deformaciones infinitesimales para las superficies $F_{1}^{+}, F^{2}, F^{+}$. Para $F_{1}^{+}$vamos tener:

$$
\begin{array}{ll}
\text { 1) } & \delta \tilde{\omega}_{1}^{+1} \wedge \tilde{\omega}_{2}^{+1}+\tilde{\omega}_{1}^{+1} \wedge \delta \tilde{\omega}_{2}^{+1}=0, \\
\text { 2) } & d \delta \tilde{\omega}_{1}^{+1}=\phi_{1}^{+} \wedge \delta \tilde{\omega}_{2}^{+1} \\
\text { 3) } & d \delta \tilde{\omega}_{2}^{+1}=\phi_{2}^{+1} \wedge \delta \tilde{\omega}_{1}^{+1} \\
\text { 4) } & \tau^{+1} \wedge \delta \tilde{\omega}_{1}^{+1}+\tau^{+2} \wedge \delta \tilde{\omega}_{2}^{+1}=0
\end{array}
$$

El sistema de ecuaciones para la superficie $F_{2}$ tendrá la forma:

$$
\begin{aligned}
& \text { 1) } \delta \tilde{\omega}_{3}^{3} \wedge \omega_{4}^{3}+\omega_{3}^{3} \wedge \delta \tilde{\omega}_{4}^{3}=0, \\
& \text { 2) } d \delta \tilde{\omega}_{3}^{3}=\phi_{3}^{4} \wedge \delta \tilde{\omega}_{4}^{3}, \\
& \text { 3) } d \delta \tilde{\omega}_{4}^{3}=\phi_{4}^{3} \wedge \delta \tilde{\omega}_{3}^{3} \\
& \text { 4) } \tau^{3} \wedge \delta \tilde{\omega}_{3}^{3}+\tau^{4} \wedge \delta \tilde{\omega}_{4}^{3}=0 .
\end{aligned}
$$

Teniendo en cuenta que las formas de inmersión y las formas de conexión de las superficies $F_{1}^{+}$y $F^{2}$ coinciden con las formas de inmersión y formas de conexión de la superficie $F^{+}$, el principal sistema de deformaciones infinitesimales isométricas para la superficie $F^{+}$, lo escribiremos de la siguiente forma: 
1) $\delta \omega^{+1} \wedge \omega_{2}^{+1}+\omega_{1}^{+1} \wedge \delta \omega_{1}^{+1}=0$,

2) $\delta \omega^{+3} \wedge \omega_{3}^{3}+\omega^{+1} \wedge \delta \omega^{+1}{ }_{3}=0$,

3) $\delta \omega^{+3}{ }_{1}^{3} \wedge \omega_{4}^{3}+\omega^{+1} \wedge \delta \omega^{+1}=0$,

4) $\delta \omega_{2}^{+3} \wedge \omega_{3}^{3}+\omega_{2}^{+1} \wedge \delta \omega_{3}^{+1}=0$,

5) $\delta \omega_{2}^{+3} \wedge \omega_{4}^{3}+\omega_{2}^{+1} \wedge \delta \omega_{4}^{+1}=0$,

6) $\delta \omega^{+}{ }_{3}^{3} \wedge \omega_{4}^{3}+\omega_{3}^{+3} \wedge \delta \omega^{+3}{ }_{4}^{3}=0$,

7) $d \delta \omega_{1}^{+1}=\phi_{1}^{+2} \wedge \delta \omega_{2}^{+1}$,

8) $\quad d \delta \omega_{2}^{+1}=\phi_{2}^{+1} \wedge \delta \omega_{1}^{+1}$,

9) $\quad d \delta \omega^{+1}{ }_{3}=\phi_{3}^{4} \wedge \delta \omega^{+1}+\omega_{3}^{3} \wedge \delta \varkappa_{3}^{+1}$,

10) $d \delta \omega^{+1}=\phi_{4}^{3} \wedge \delta \omega_{3}^{+1}+\omega_{4}^{3} \wedge \delta \varkappa_{3}^{+1}$,

11) $d \delta \omega^{+3}=\phi_{1}^{+2} \wedge \delta \omega_{2}^{+3}+\omega^{+1} \wedge \delta \varkappa_{1}^{+3}$,

12) $d \delta \omega_{2}^{+3}=\phi_{2}^{+1} \wedge \delta \omega^{+3}+\omega_{2}^{+1} \wedge \delta \varkappa_{1}^{+3}$,

13) $d \delta \omega^{+3}{ }_{3}^{3}=\phi_{3}^{4} \wedge \delta \omega_{4}^{+3}$,

14) $d \delta \omega_{4}^{+3}=\phi_{4}^{3} \wedge \delta \omega_{3}^{+3}$,

15) $\tau^{+1} \wedge \delta \omega_{1}^{+1}+\tau^{+^{2}} \wedge \delta \omega_{2}^{+1}+\tau^{3} \wedge \delta \omega_{3}^{+1}+\tau^{4} \wedge \delta \omega_{4}^{+1}=0$,

16) $\tau^{+1} \wedge \delta \omega_{1}^{+3}+\tau^{+^{2}} \wedge \delta \omega_{2}^{+3}+\tau^{3} \wedge \delta \omega_{3}^{+3}+\tau^{4} \wedge \delta \omega_{4}^{+3}=0$,

17) $d \delta \varkappa_{3}^{+1}=\delta \omega_{3}^{+1} \wedge \omega_{3}^{3}+\delta \omega^{+1} \wedge \omega_{4}^{3}+$ $+\omega^{+1} \wedge \delta \omega_{1}^{+3}+\omega_{2}^{+1} \wedge \delta \omega_{2}^{+3}$.

2.5.3.A. Consideremos las ecuaciones 2) - 5) de este sistema. Multipliquemos las ecuaciones 2) y 4) de forma externa por $\omega_{3}^{3}$. En resumen obtenemos:

$$
\begin{aligned}
& \omega_{3}^{3} \wedge \omega_{1}^{+1} \wedge \delta \omega_{3}^{+1}=0, \\
& \omega_{3}^{3} \wedge \omega_{2}^{+1} \wedge \delta \omega_{3}^{+1}=0 .
\end{aligned}
$$

De aquí surge que la forma $\delta \omega^{+1}$, se puede representar en forma de combinación lineal:

$$
\begin{aligned}
& \delta \omega_{3}^{+1}=\lambda \omega_{1}^{+1}+\mu \omega_{3}^{3}, \\
& \delta \omega_{3}^{+1}=\alpha \omega_{2}^{+1}+\beta \omega_{3}^{3},
\end{aligned}
$$

donde $\lambda, \mu, \alpha, \beta$ son algunas funciones.

Restando una ecuación de otra, vamos a tener:

$$
\lambda \omega_{1}^{+1}-\alpha \omega_{2}^{+1}+(\mu-\beta) \omega_{3}^{+3}=0 .
$$

De la independencia lineal de las formas, que forman parte de esta ecuación, surge que $\lambda=0, \alpha=0, \mu=\beta$ y la forma $\delta \omega^{+1}$, se escribe de la siguiente manera:

$$
\delta \omega_{3}^{+1}=\mu \omega_{3}^{3} .
$$

De forma analógica, usamos los mismos criterios de la 2) y 3) para la forma $\delta \omega^{+3}{ }_{1}^{3}$ obteniendo:

$$
\delta \omega_{1}^{+3}=\rho \omega_{1}^{+1},
$$


y de las 3), 5) y 4), 5) para las formas $\delta \omega^{+}{ }_{4}^{1} \mathrm{y} \delta \omega^{+3}{ }_{2}$ encontramos:

$$
\begin{aligned}
& \delta \omega_{4}^{+1}=\xi \omega_{4}^{3}, \\
& \delta \omega_{2}^{+3}=\gamma \omega_{2}^{+1},
\end{aligned}
$$

donde $\rho, \xi, \gamma$ son algunas funciones.

Sustituyendo las expresiones obtenidas en las ecuaciones 2), 3), 4), llegamos a las igualdades:

$$
\begin{aligned}
& (\rho+\mu) \omega_{1}^{+1} \wedge \omega_{3}^{3}=0, \\
& (\rho+\xi) \omega^{+}{ }_{1}^{1} \wedge \omega_{4}^{3}=0, \\
& (\gamma+\mu) \omega_{2}^{+1} \wedge \omega_{3}^{3}=0,
\end{aligned}
$$

de aquí surge que $\rho=\gamma=-\mu, \xi=\mu$.

De tal forma, para $\delta \omega_{3}^{+1}, \delta \omega_{1}^{+3}, \delta \omega^{+}{ }_{4}^{1}, \delta \omega^{+3}{ }_{2}$ tenemos:

$$
\begin{aligned}
& \delta \omega_{3}^{+1}=\mu \omega_{3}^{3}, \\
& \delta \omega_{1}^{+3}=-\mu \omega_{1}^{+1}, \\
& \delta \omega_{4}^{+1}=\mu \omega_{4}^{3}, \\
& \delta \omega_{2}^{+3}=-\mu \omega_{2}^{+1} .
\end{aligned}
$$

La variación de las formas de inmersión de este tipo, corresponden a la variación de las deformaciones infinitesimales isométricas triviales de la superficie $F^{+} \subset E^{6}$ (ver [4], pág. 76)

2.5.3.B. Sustituyendo (16), en las ecuaciones 15) y 16) del sistema (15), utilizando (4) obtenemos:

$$
\begin{aligned}
& \tau^{+1} \wedge \delta \omega_{1}^{+1}+\tau^{+2} \wedge \delta \omega_{2}^{+1}=0 \\
& \tau^{3} \wedge \delta \omega_{3}^{+3}+\tau^{4} \wedge \delta \omega_{4}^{+3}=0
\end{aligned}
$$

Observemos que estas ecuaciones, en conjunto con las ecuaciones 1), 6), 7), 8), 13), 14) del sistema (15), coinciden con las ecuaciones de los sistemas (13) y (14). En vista de la condición del teorema las superficies $F_{1}$ y $F_{2}$ son rígidas en $E_{1}^{3}$ y $E_{2}^{3}$ respectivamente, entonces las soluciones de los sistemas (13) y (14), corresponden a las de las deformaciones infinitesimales isométricas triviales de las superficies $F_{1}$ y $F_{2}$, o sea, son nulas [3]. Por consiguiente, $\delta \omega_{1}^{+1}=\delta \omega_{2}^{-1}=\delta \omega_{3}^{+3}=\delta \omega^{+3}=0$.

2.5.2.C. Consideremos las ecuaciones 11) y 12) de sistema (15). Usando (16), llegamos a las igualdades:

$$
\begin{aligned}
& -d \mu \wedge \omega_{1}^{+1}-\mu d \omega_{1}^{+1}=\phi_{2}^{+1} \wedge \mu \omega_{2}^{+1}-\omega_{1}^{+1} \wedge \delta \varkappa_{3}^{+1}, \\
& -d \mu \wedge \omega_{2}^{+1}-\mu d \omega_{2}^{+1}=-\phi_{2}^{+1} \wedge \mu \omega_{1}^{+1}-\omega_{2}^{+1} \wedge \delta \varkappa_{3}^{+1} .
\end{aligned}
$$


De aquí, con la utilización de las ecuaciones de Peterson-Codazzi obtenemos:

$$
\begin{aligned}
& \left(d \mu+\delta \varkappa_{3}^{+1}\right) \wedge \omega_{1}^{+1}=0 \\
& \left(d \mu+\delta \varkappa_{3}^{+1}\right) \wedge \omega_{2}^{+1}=0 .
\end{aligned}
$$

De tal manera, la forma $d \mu+\delta \varkappa_{3}^{+1}$ se puede representar así:

$$
d \mu+\delta \varkappa_{3}^{+1}=a \omega^{+1},
$$

donde $a$ es alguna función. Sustituyendo esta expresión en la segunda igualdad de (17), aprovechando la ecuación de Gauss, vamos a tener:

$$
a K \tau^{+1} \wedge \tau^{+^{2}}=0
$$

De aquí surge que $a=0$.

Para $\delta \varkappa_{3}^{+1}$ de la (18), obtenemos:

$$
\delta \varkappa_{3}^{+1}=-d \mu .
$$

Este tipo de $\delta \varkappa^{+1}$ corresponde al de las deformaciones infinitesimales isométricas triviales de la superficie $F^{+}$(ver [3]).

De tal manera que en las superficies $F^{+}$y $F^{-}$, existen sólo campos de deformaciones isométricas triviales, o sea, campos del tipo $\delta \mathbf{r}^{+}=\Omega^{+} \cdot \mathbf{r}+\omega^{+}$y $\delta \mathbf{r}^{-}=\Omega^{-} \cdot \mathbf{r}+\omega^{-}$. En el punto 2.5.2 se demostró que $\mathbf{V}^{+}=\mathbf{V}^{-}$en la superficie de pegado $S$. De esto y de la (6), surge que $\mathbf{V}^{+}=\mathbf{V}^{-}=\Omega^{+}=\Omega^{-}$. De la condición $\delta \mathbf{r}^{+}(x)=\delta \mathbf{r}^{-}(x)$ en $\Gamma$, obtenemos del mismo modo que $\omega^{+}=\omega^{-}$y esto significa la trivialidad del campo de deformaciones isométricas en la superficie $F^{=} F^{+} \cup F^{-}$.

\section{Referencias}

[1] Markov P. E. (1980) "Deformaciones isométricas infinitesimales de algunas superficies multidimensionales", Matem. zametki 27 (3):469-479.

[2] Markov P. E. (1997) "Deformaciones infinitesimales y analíticas generales de una inmersión 1", Izv. Vusov. Matemática 9:21-34.

[3] Markov P. E. (1997) "Deformaciones infinitesimales y analíticas generales de una inmersión 2", Izv. Vusov. Matemática 9:41-51.

[4] Markov P. E. (2001) "Número típico y rigidez de superficies fibradas", Matem. sbornik 192(1):67-88. 